\title{
Drinking water prior to a meal does not affect hunger and satiety ratings in young adults and older subjects
}

\author{
M. Sayegh and B. Ratcliffe \\ Robert Gordon University, St Andrew Street, Aberdeen, AB25 1HG, UK
}

Tactics to address dietary overconsumption include reducing the energy density of meals on the assumption that eating for volume or mass leads to lower energy intake ${ }^{(1)}$. This predicates the absence of compensatory mechanisms to maintain energy intake in the longer term. There is certainly evidence that energy density is positively associated with weight gain ${ }^{(2)}$ but it is less clear that manipulating energy density leads to long term weight loss or improved weight maintenance. Some research has focused on enhancing satiety to reduce energy intake at meals ${ }^{(3)}$. Studies of test meals in laboratory conditions showed that water consumed with a meal reduced ratings of hunger and increased ratings of satiety ${ }^{(4,5)}$. The effect of drinking water before a meal produced variable effects depending on age ${ }^{(6)}$. However, there is a lack of similar free-living studies, so the aim of this study was to compare the effects of water preload on satiety ratings and fullness with those of no water preload in two age groups in free living conditions.

There were ten young and four older subjects with a mean age of 26 (SD 8.2) and 56 (SD 5.3) years, respectively. Subjects chose two days in a typical week and recorded their main meals on those days in a food diary. In the second week, they were asked to use the same two days and have the same main meals but they were required to drink $500 \mathrm{ml}$ of water as a preload in the $15 \mathrm{~min}$ before the main meal. Subjective appetite ratings were determined using visual analogue scales (VAS) before the meal (and the preload), immediately after, and 90 min after in both weeks. The table shows median VAS (mm) and inter-quartile range (IQR) for all subjects $(n$ 14).

\begin{tabular}{|c|c|c|c|c|c|c|c|c|c|c|c|c|}
\hline \multirow[b]{2}{*}{ Ratings } & \multicolumn{6}{|c|}{ Week 1 (No preload) } & \multicolumn{6}{|c|}{ Week 2 (Water preload) } \\
\hline & Pre-meal & IQR & After meal & IQR & 90 min after & $\overline{\mathrm{IQR}}$ & Pre-meal & IQR & After meal & IQR & 90 min after & $\overline{\mathrm{IQR}}$ \\
\hline Hunger & 67.9 & 41.56 & 6.1 & 7.06 & 21.6 & 26.56 & 72.9 & 23.25 & 4.5 & 4.69 & 19.6 & 30.88 \\
\hline Satiety & 29.1 & 21.13 & 89.0 & 17.75 & 80.8 & 23.00 & 22.0 & 24.88 & 88.4 & 12.75 & 65.1 & 26.88 \\
\hline Fullness & 33.5 & 22.00 & 89.6 & 10.88 & 77.8 & 21.63 & 22.1 & 29.19 & 92.6 & 10.50 & 76.8 & 23.69 \\
\hline Prospective consumption & 71.9 & 25.00 & 8.8 & 12.13 & 29.0 & 22.00 & 76.8 & 23.69 & 5.0 & 9.50 & 19.4 & 26.00 \\
\hline Desire for sweet & 18.4 & 35.13 & 44.4 & 51.00 & 24.6 & 57.00 & 17.8 & 32.88 & 16.4 & 50.06 & 43.5 & 51.44 \\
\hline Desire for savoury & 78.6 & 29.69 & 6.4 & 13.44 & 13.1 & 29.00 & 84.4 & 27.25 & 2.5 & 8.75 & 12.0 & 26.31 \\
\hline
\end{tabular}

Using multiple comparisons and post hoc tests meant that $P<0.016$ was required for significance. There were no differences between weeks for the pre-meal conditions, as expected and desirable, and no differences between the age groups but also there was no effect of the pre-load on subsequent ratings either measured in absolute terms or as differences from the pre-meal values.

The study did not lend itself to a cross-over design and there were limited opportunities for checks on compliance with the protocol. Nevertheless, it is concluded that drinking water prior to a meal does not affect subjective measures of satiety. However, energy intake was not measured and satiety may have been achieved at lower intakes following the preload.

1. Bell EA, Castellanos VH, Pelkman CL et al. (1998) Am J Clin Nutr 67, 412-420.

2. Savage JS, Marini M \& Birch LL (2008) Am J Clin Nutr 88, 677-684.

3. Swinburn BA, Caterson I, Seidell JC et al. (2004) Public Health Nutr 7, 123-146.

4. Lappalainen R, Mennen L, van Weert L et al. (1993) Eur J Clin Nutr 47, 815-819.

5. DellaValle DM, Roe LS \& Rolls BJ (2005) Appetite 44, 187-193.

6. Van Walleghen EL, Orr JS, Gentile CL et al. (2007) Obesity 15, 93-99. 